\title{
Nucleation and supersaturation in porous media (revisited)
}

\author{
M. PRIETO
}

Department of Geology, University of Oviedo, 33005-Oviedo, Spain

[Received 21 May 2014; Accepted 9 November 2014; Associate Editor: T. Stawski]

\section{ABSTRACT}

Supersaturation-Nucleation-Time (S-N-T) diagrams are shown to be a useful tool to predict nucleation during reactive-transport processes in porous media. Such diagrams can be determined experimentally or estimated from theoretical calculations based on classical nucleation theory. With this aim, a 'pragmatic' understanding of the nucleation rate equation is adopted here and the meaning and magnitude of the interfacial tension and induction time discussed. Theoretical diagrams and experimental data are shown to match fairly well as long as there is an appropriate choice of the 'relevant' volume for induction-time calculations.

KEYWORDs: nucleation, supersaturation, porous media.

\section{Introduction}

NUCLEATION is in the scientific spotlight, as shown by the number of reviews published on the subject in the last few years, particularly in relation to crystal nucleation in solution (Benning and Waychunas, 2008; Vekilov, 2010; Yi and Rutledge, 2012; Gebauer et al., 2014). These additions to the literature are no doubt related to the detection of stable solute species, referred to as pre-nucleation clusters (PNCs), which are supposed to mediate the development of the crystal phase from the aqueous solution (Gebauer et al., 2008; Gebauer and Cölfen, 2011). That breakthrough challenges our idea of the mechanisms involved in the nucleation process as defined in classical nucleation theory (CNT). The use of state-of-the-art techniques (Pouget et al., 2009) and molecular simulation methods (Demichelis et al., 2011) reveals the nature of PNCs and making the statement "now you see them" (Meldrum and Sear, 2008) convincing, at least in the case of calcium carbonate. Other inorganic systems such as certain iron oxy-hydroxides (e.g. Yuwono et al., 2010), calcium phosphates (e.g. Wang et al.,

*E-mail: mprieto@geol.uniovi.es

DOI: $10.1180 /$ minmag.2014.078.6.11
2012), and silica could be reasonably accommodated in the new nucleation paradigm, although the recognition as PNCs of certain oligomers, amorphous nano-clusters, and other "primary" particles is a source of controversy (Gebauer et al., 2014). Moreover, the stability of many of these species and their effect on nucleation barriers remains a point for discussion (De Yoreo, 2013). Finally, even if 'primary' particles exist in a given aqueous system, the formation of precursor phases by aggregation may not occur (Baumgartner et al., 2013), and the process can still be described within the CNT framework.

Much work needs to be done to extend the aggregation-based mechanism to inorganic systems other than $\mathrm{CaCO}_{3}$, and therefore nucleation will continue to be a leading topic in the years to come. In the meantime, kinetic modelling of nucleation is typically performed using the classical nucleation rate equation, with some exceptions in systems that are known to involve amorphous precursor phases (e.g. RodríguezBlanco et al., 2011). CNT is used not only in

This paper is published as part of a special issue in Mineralogical Magazine, Vol. 78(6), 2014 entitled 'Mineral-fluid interactions: scaling, surface reactivity and natural systems'. 
industrial crystallization protocols (Sangwal, 2007) but also in geochemical modelling (Fritz and Noguera, 2009) and in fundamental studies of precipitation and co-precipitation on mineral surfaces (Fernández-Martínez et al., 2013; Shtukenberg et al., 2005).

In CNT, the nucleation rate (the number of nuclei, $N$, formed per unit volume and time: $J=$ $N / V t$ ) depends exponentially on the free energy change $\left(\Delta G_{\mathrm{c}}\right)$ involved in the formation of a nucleus of critical size, i.e.

$$
J=\Gamma \exp \left(-\frac{\Delta G_{\mathrm{c}}}{\mathrm{k} T}\right)
$$

where $\mathrm{k}$ is the Boltzmann constant, $T$ the absolute temperature, and $\Gamma$ a pre-exponential factor. In turn, $\Delta G_{\mathrm{c}}$ depends significantly on the supersaturation $(S)$, according to

$$
\Delta G_{\mathrm{c}}=\frac{\beta \omega^{2} \sigma^{3}}{(\mathrm{k} T \ln S)^{2}}
$$

where $\omega$ is the molecular volume in the solid phase, $\beta$ is a factor that depends on the shape of the nucleus, and $\sigma$ is the interfacial tension. Most readers are likely to be aware of the weak points implicit in CNT, such as the idea of applying macroscopic concepts (surface and volume) to molecular-scale objects or the assumption that the interfacial tension does not depend on the size of the nuclei. While there is a general agreement on these weak points, the method by which the controlling parameters in equations 1 and 2 are estimated is widely disregarded. For example, the interfacial tension is recognized to represent all the excess energy accumulated in the vicinity of the solution-cluster interface, but in practice, $\sigma$ is an 'artificial' parameter that cannot be determined by direct and unambiguous experiments (Sönhel, 1982). In fact, the opposite approach must be adopted: the only interfacial tension values that are relevant in modelling nucleation kinetics are those obtained in nucleation experiments, i.e. from nucleation-rate or induction-time measurements. Although the term 'interfacial tension' is used interchangeably in the literature, the nucleationderived $\sigma$ has a different meaning and value from the "surface energies" obtained by contact-angle measurements (Wu and Nancollas, 1999) or wateradsorption calorimetry (Forbes et al., 2011). Moreover, the CNT-derived $\sigma$ values depend on the aqueous speciation model, the expression chosen for the supersaturation, and the shape factor chosen for the nuclei (Prieto et al., 2012). This fact was well known by the pioneers in this field (Söhnel, 1982) but is frequently disregarded in the literature. Therefore, to model the nucleation kinetics in a given system, all the input data must be consistent with the value of $\sigma$ chosen for the calculation. Thus, the CNT expression in equation 1 can be imagined as a species of fitting function in which the main fitting parameter is $\sigma$. The nucleation barrier depends heavily $\left(\Delta G_{\mathrm{c}} \propto \sigma^{3}\right)$ on this empirical magnitude, whereas other less influential parameters (particularly those included in the pre-exponential factor) can be tuned to optimize the results. From that point of view, $\Delta G_{\mathrm{c}}$ plays a similar role to the energy barriers to form activated complexes, which are typical in chemical kinetics. Moreover, phenomena such as the singular nucleation behaviour of sparingly soluble compounds in ionic solutions (Kowacz et al., 2010) could be modelled by CNT, provided that ad hoc nucleation-derived $\sigma$ data were available.

According to this pragmatic understanding of CNT, the present study models the nucleation behaviour observed in previous experiments (Putnis et al., 1995), taking baryte as an example system. Such experiments were conducted in U-shaped tubes in which the reacting ions counter-diffuse through a column of porous silica hydrogel. Because the gel properties inhibit advection and convection, the crystallization medium is purely diffusive. Crystals tend to nucleate approximately midway through the diffusion column, which is the zone where the supersaturation builds up most rapidly because the counter-diffusing ions 'meet' there. Hydrogel media have been used widely to simulate precipitation and reactive transport in natural environments (Fernández-Díaz et al., 1996; Prieto et al., 2002) and are currently used in bio-mineralization studies (Sancho-Tomás et al., 2013; Nindiyasari et al., 2014). In nature, precipitation frequently occurs in pores of rocks, soils and sediments. Moreover, crystallization of salts in porous building materials (masonry, cement, mortar) has been recognized as crucial for their weathering and decay (RodríguezNavarro et al., 2002). Developing predictive models for the nucleation behaviour in these types of systems is worthwhile, therefore. With this aim, we use a type of supersaturationnucleation-time diagram (S-N-T) that allows the interpretation of nucleation pathways in solution systems. S-N-T diagrams are reminiscent of the 


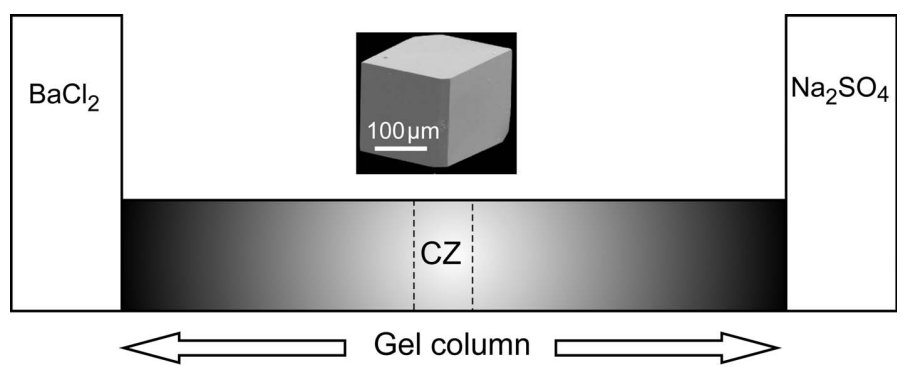

FIG. 1. Typical U-tube arrangement. The horizontal branch $(28 \mathrm{~cm})$ connects two vertical branches $(10 \mathrm{~cm})$ which contain the parent solutions. The SEM image shows a typical baryte crystal grown by this technique.

temperature-transformation-time (T-T-T) diagrams used to interpret sub-solidus transformations in mineralogy and metallurgy (Putnis, 1992) and can be used similarly. The choice of baryte as an example system is not arbitrary. Baryte-scale formation is a problem in many industrial processes where knowledge of the nucleation kinetics is essential for developing anti-scale strategies. However, while most precipitation studies have been performed using vigorously stirred solutions, in many scenarios the nucleation of baryte occurs in pores or small cavities where the confined solution can become more highly supersaturated than an analogous free solution (Putnis and Mauthe, 2001; Rodríguez-Ruiz et al., 2014). The present study confirms that hydrogels are excellent media to emulate nucleation of minerals in such conditions of confinement.

\section{Methods}

\section{Precipitation experiments}

The experiments addressed here were described broadly in the original papers by Putnis et al.
(1995) and Prieto et al. (1990). The U-tube arrangement in which two solution reservoirs are separated by a diffusion column of silica hydrogel is shown in Fig. 1. The gel contains $~ 95.6$ vol.\% solution within interconnecting pores of diameters $0.1-0.5 \mu \mathrm{m}$. However, the pore size is not uniform (Henisch, 1988) and secondary pores with diameters $>10 \mu \mathrm{m}$ are common. The initial gel $\mathrm{pH}$ is 5.5 , and the working temperature is $25^{\circ} \mathrm{C}$. In such a device, the experimental inputs are the Utube dimensions and the initial concentration of the reactants, while the primary outputs are the location of the crystallization zone (cz) and the time $\left(t_{\mathrm{w}}\right)$ at which the first crystallites are observed by optical microscopy at $\times 500$ magnification. These data, as provided by Putnis et al. (1995) and related papers, are shown in Table 1. The secondary parameters have been recalculated here to make them consistent with the overall calculation procedure. Solution speciation, masstransfer, and supersaturation have been computed using the geochemical code PHREEQC (Parkhurst and Appelo, 2013) and its default database. PHREEQC allows modelling of one-dimensional

TABLE 1. Experimental data

\begin{tabular}{|c|c|c|c|c|c|c|c|}
\hline \multirow[t]{2}{*}{ Code } & \multicolumn{2}{|c|}{ Parent solutions (M) } & \multirow{2}{*}{$\begin{array}{c}t_{\mathrm{w}} \\
(\mathrm{h} \pm 6)\end{array}$} & \multirow{2}{*}{$\begin{array}{c}\mathrm{cz} \\
(\mathrm{cm} \pm 1)\end{array}$} & \multirow{2}{*}{$\begin{array}{l}\Omega^{\text {th }} \\
\times 10^{3}\end{array}$} & \multirow{2}{*}{$\begin{array}{c}R_{\Omega} \\
\left(\mathrm{h}^{-1}\right)\end{array}$} & \multirow{2}{*}{$\begin{array}{c}t_{\mathrm{R}} \\
(\mathrm{h})\end{array}$} \\
\hline & $\mathrm{BaCl}_{2}$ & $\mathrm{Na}_{2} \mathrm{SO}_{4}$ & & & & & \\
\hline BRT-1 & 0.5 & 0.5 & $380 *$ & 14 & $11.9 \pm 1.2$ & 190 & 62.6 \\
\hline BRT-2 & 0.5 & 0.3 & 432 & 16 & $10.7 \pm 0.9$ & 144 & 74.1 \\
\hline BRT-3 & 0.3 & 0.5 & 458 & 14 & $11.5 \pm 0.9$ & 136 & 84.3 \\
\hline BRT-4 & 0.5 & 0.1 & 476 & 18 & $7.30 \pm 0.6$ & 90 & 81.1 \\
\hline BRT-5 & 0.3 & 0.3 & 480 & 15 & $8.18 \pm 1.5$ & 94 & 87.1 \\
\hline BRT-6 & 0.1 & 0.5 & 504 & 13 & $8.11 \pm 0.5$ & 80 & 101.4 \\
\hline BRT-7 & 0.1 & 0.1 & 572 & 15 & $4.06 \pm 0.3$ & 35 & 116.1 \\
\hline
\end{tabular}

* Note that in Prieto et al. (1990) and Putnis et al. (1995) this value was mistakenly compiled as $360 \mathrm{~h}$. 
diffusion with the same algorithm that was used by Henisch and García-Ruiz (1986) to model the diffusion and precipitation patterns in gel columns. The newly obtained parameters $\left(\Omega^{\text {th }}\right.$ and $\left.R_{\Omega}\right)$ differ from the original ones but correlate similarly with each other.

For sparingly soluble minerals, the thermodynamic supersaturation is given by the quotient between the ionic activity product in the current solution and the thermodynamic solubility product. In the case of baryte:

$$
\Omega=\left\{\mathrm{Ba}^{2+}\right\} \cdot\left\{\mathrm{SO}_{4}^{2-}\right\} / \mathrm{K}_{\mathrm{Brt}}
$$

In Table $1, \Omega^{\text {th }}$ represents the value of $\Omega$ at $t_{\mathrm{w}}$, and column $R_{\Omega}$ lists the supersaturation rate $(d \Omega / d t)$ at $t_{\mathrm{w}}$. The waiting time $t_{\mathrm{w}}$ can be considered as representative of nucleation: once a crystal nucleates, the growth to reach a visible size $(\times 500)$ is very fast due to the high supersaturation level in the nucleation area (Table 1). Even so, the imprecision in determining the nucleation event can be expected to depend on the observer's care and thoroughness. Here, we have chosen deliberately an overestimated value of $\pm 6 \mathrm{~h}$, considering a $3 \mathrm{~h}$ monitoring interval. This imprecision is not very significant $(<2 \%)$ when compared with the $t_{\mathrm{w}}$ values but needs to be incorporated in the calculations. The last column in Table $1\left(t_{\mathrm{R}}\right)$ represents the time elapsed to reach $\Omega^{\text {th }}$ at a constant rate equal to $R_{\Omega}$.

\section{CNT calculations}

There are numerous compilations of CNT-derived $\sigma$ values in the literature, but most of them come from the same original papers by Nielsen (1967), Garten and Head (1973) and a small number of others. These original data were determined using concentrations instead of activities to express the supersaturation or simply introducing correction factors for the degree of dissociation. Here, to use a value consistent with the PHREEQC code, $\sigma$ has been recalculated from some of these original data. Garten and Head (1973) determined a value of $107 \mathrm{~mJ} / \mathrm{m}^{2}$ considering spherical nuclei $(\beta=$ $16 \pi / 3)$ and a supersaturation scale $\left(\mathrm{s}_{ \pm}\right)$) based on mean ionic activities $\left(a_{ \pm}\right)$in the aqueous solution, where $a_{ \pm}=\left(a_{+}^{v+} a_{-}^{v-}\right)^{1 / v}$, and $v=v_{+}+v_{-}$is the number of ions in the solute formula. In contrast, Nielsen (1967) determined a value of $135 \mathrm{~mJ} / \mathrm{m}^{2}$ using an $\Omega$ scale and cubic nuclei $(\beta=32)$. We can convert the Garten and Head $(\mathrm{G} \& \mathrm{H})$ value to Nielsen's scale using the expression:

$$
\sigma(\text { Neilsen })=\sigma(\mathrm{G \& H}) \cdot v^{2 / 3} \cdot \pi(\pi / 6)^{1 / 3}
$$

On the basis of Nielsen, the interfacial tension obtained by G\&H is converted to $137 \mathrm{~mJ} / \mathrm{m}^{2}(v=$ 2 for baryte), a value very close to Nielsen's result, which confirms the consistency of both data sets. Starting from the experimental data by G\&H, a value of $134 \mathrm{~mJ} / \mathrm{m}^{2}$ has been recalculated using an $\Omega$-PHREEQC scale and spherical nuclei. Note that, despite the appearance, this value differs significantly from Nielsen's, which was given for cubic nuclei. The spherical approximation is physically reasonable for the size range considered, in which the particles occurring in many processes tend to be spherical (Liu, 1999).

In CNT, the pre-exponential factor is also a key term that represents the rate of attachment of monomers (growth units or molecules) to the critical nuclei. Different approximations to derive this factor can be seen in the literature (Walton, 1969; Lasaga, 1998; Kashchiev, 2000; Sangwal, 2007). When the attachment is controlled by the diffusion of growth units from the solution bulk to the nucleus surface, a comprehensive formulation (Kashchiev, 2000) results in:

$$
\Gamma=Z \frac{D N_{1}}{r_{\mathrm{c}}} A_{\mathrm{c}} N_{0}
$$

where $N_{0}$ and $N_{1}$ are concentrations that represent the number of nucleation sites and the number of monomers per unit volume of fluid, respectively. $D$ is the diffusion coefficient of the monomers in the fluid phase, and $A_{\mathrm{c}}$ is the surface area of the critical nucleus, assumed to be a sphere of radius $r_{\mathrm{c}}$ (with $\left.r_{\mathrm{c}}=2 \omega \sigma / \mathrm{k} T \ln \Omega\right)$. The term $D N_{1} / r_{\mathrm{c}}$ stands for the incoming diffusion flux of monomers to the nucleus surface. Finally, the Zeldovich factor $(Z)$ arises from the steady-state treatment of the problem:

$$
Z=\left(\frac{\Delta G_{\mathrm{c}}}{3 \pi \mathrm{k} T n_{\mathrm{c}}^{2}}\right)^{\frac{1}{2}}
$$

where $n_{\mathrm{c}}$ is the number of monomers in the critical nucleus. For homogeneous (HON) nucleation, every molecular position can be considered a potential nucleation site (Kashchiev and Rosmalen, 2003). Therefore, $N_{0}$ can be equated to $1 / v_{\mathrm{w}}$, where $v_{\mathrm{w}}$ is the volume of a water molecule in the solution. The calculations were implemented in Mathcad (MathSoft Inc.) using the parameters compiled in Table 2. The concentration of monomers $\left(N_{1}\right)$ depends on the super- 


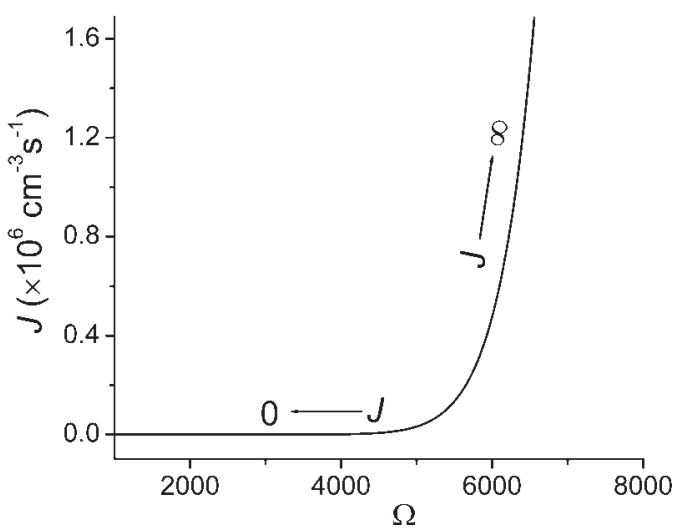

FIG. 2. The nucleation rate of baryte as a function of supersaturation. The calculation parameters are shown in Table 2 (row HON).

saturation and was calculated in each case using PHREEQC. Simulations of 3D-heterogeneous nucleation (HEN) on foreign micro-particles have been performed using a unified operational formula in which HON is treated as a limiting case of HEN with $\sigma$ replaced by an effective interfacial tension defined as:

$$
\sigma_{\text {eff }}=\Phi \sigma
$$

where the coefficient $\Phi$ is a number between 0 and 1 (Sangwal, 2007; Liu, 1999) that depends on both the substrate-nucleus interaction and the geometry of the assemblage.

\section{Calculation results and discussion \\ Critical supersaturation and threshold supersaturation}

The classical nucleation rate equation is based on the assumption that supersaturation is reached instantaneously. For a given supersaturation, the nucleation rate adopts a specific value, and we can represent a function that relates the two parameters. Figure 2 represents such a function for baryte. As shown, the nucleation rate increases so dramatically with supersaturation that the occurrence of a critical value is usually admitted. Above this value, the nucleation becomes catastrophic $(J \rightarrow \infty)$, and below this value, nucleation decreases quickly to zero. The critical supersaturation is considered to be representative of the metastability limit, which marks the width of the so-called metastable zone. However, the critical supersaturation needs to be defined by choosing an arbitrary value of $J$ (typically $1 \mathrm{~s}^{-1} \mathrm{~cm}^{-3}$ ), and therefore, the metastability limit is ambiguous. Moreover, in most scenarios, supersaturation is not reached instantaneously but increases continuously until nucleation (whether HEN or HON) occurs. That phenomenon also occurs in counter-diffusion experiments, for which the concept of threshold supersaturation was first defined (Putnis et al., 1995; Prieto et al., 1990). The threshold supersaturation, $\Omega^{\text {th }}$, is the effective supersaturation at which nucleation occurs and has been shown to depend on the reaction path followed by the system.

Figure 3 shows the increase in supersaturation in $\mathrm{cz}$ as a function of time in the case of experiment BRT-1 (see Table 1 also). The highest value is $\Omega^{\text {th }}$. The supersaturation increases to reach an almost constant value of $R_{\Omega}$. The inset shows at a larger scale that there is a linear trend for long diffusion times. The supersaturation rate, $R_{\Omega}$, is given by the slope of this line. Figure 3 also shows $t_{\mathrm{R}}$, i.e. the waiting time when supersaturation increases from zero to $\Omega^{\text {th }}$ at a constant rate equal to $R_{\Omega}$. According to the experiments of Putnis et al. (1995), inspection of Table 1 shows

TABLE 2. Nucleation parameters.

\begin{tabular}{lccccccc}
\hline Mechanism & $\sigma_{\text {eff }}\left(\mathrm{J} \mathrm{cm}^{-2}\right)$ & $\Phi$ & $D\left(\mathrm{~cm}^{2} \mathrm{~s}^{-1}\right)$ & $\omega\left(\mathrm{cm}^{3}\right)$ & $N_{0}\left(\mathrm{~cm}^{-3}\right)$ & $\beta$ & $V\left(\mathrm{~cm}^{3}\right)$ \\
\hline HON & $1.34 \times 10^{-5}$ & 1 & $9.37 \times 10^{-6}$ & $8.6 \times 10^{-23}$ & $3.33 \times 10^{22}$ & $16 \pi / 3$ & 1.91 \\
HEN & $1.06 \times 10^{-5}$ & 0.79 & $9.37 \times 10^{-6}$ & $8.6 \times 10^{-23}$ & $2.50 \times 10^{7}$ & $16 \pi / 3$ & 1.91 \\
HON-0.1 $\mu \mathrm{m}$ & $1.34 \times 10^{-5}$ & 1 & $9.3 \times 10^{-6}$ & $8.65 \times 10^{-23}$ & $3.33 \times 10^{22}$ & $16 \pi / 3$ & $5 . \times 10^{-16}$ \\
HON-1 $\mu \mathrm{m}$ & $1.34 \times 10^{-5}$ & 1 & $9.3 \times 10^{-6}$ & $8.65 \times 10^{-23}$ & $3.33 \times 10^{22}$ & $16 \pi / 3$ & $5 . \times 10^{-13}$ \\
HON-100 $\mu \mathrm{m}$ & $1.34 \times 10^{-5}$ & 1 & $9.3 \times 10^{-6}$ & $8.65 \times 10^{-23}$ & $3.33 \times 10^{22}$ & $16 \pi / 3$ & $5 . \times 10^{-7}$ \\
HEN-1 $\mu \mathrm{m}$ & $9.38 \times 10^{-6}$ & 0.7 & $9.3 \times 10^{-6}$ & $8.65 \times 10^{-23}$ & $2.50 \times 10^{7}$ & $16 \pi / 3$ & $5 . \times 10^{-7}$ \\
HEN-1 $\mu \mathrm{m}$ & $1.01 \times 10^{-5}$ & 0.75 & $9.3 \times 10^{-6}$ & $8.65 \times 10^{-23}$ & $2.50 \times 10^{7}$ & $16 \pi / 3$ & $5 . \times 10^{-7}$ \\
HEN-1 $\mu \mathrm{m}$ & $1.07 \times 10^{-5}$ & 0.8 & $9.3 \times 10^{-6}$ & $8.65 \times 10^{-23}$ & $3.33 \times 10^{22}$ & $16 \pi / 3$ & $5 . \times 10^{-7}$ \\
& & & & & & & \\
\hline
\end{tabular}




\section{PRIETO}

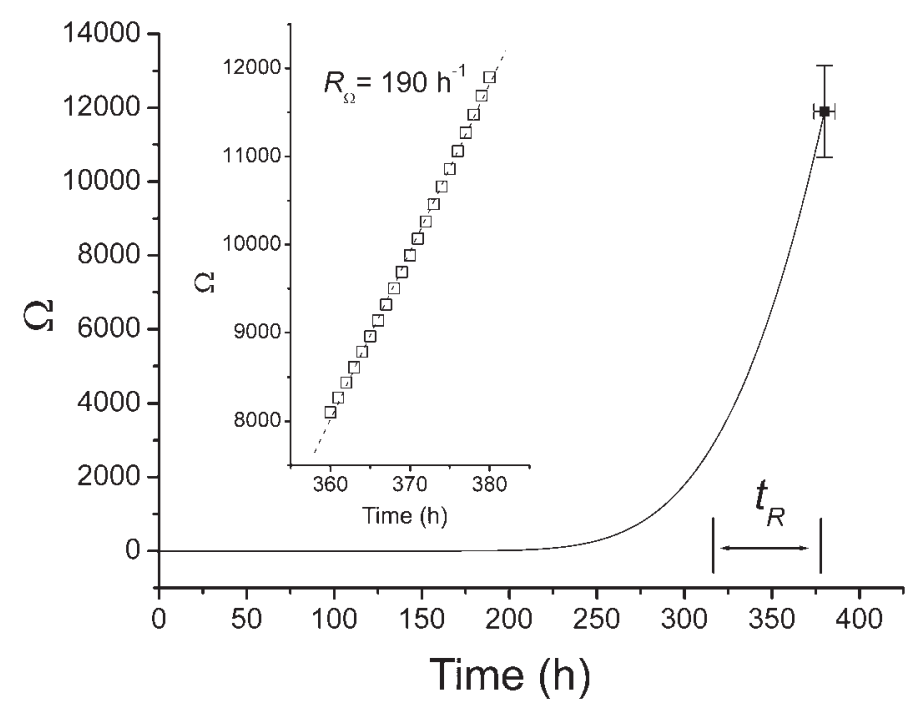

FIG. 3. Supersaturation evolution in the crystallization zone for the case of the experiment BRT-1 (see Table 1). The data-point corresponds to the threshold supersaturation. The inset shows at a larger scale that there is a linear trend for long diffusion times. The waiting time $\left(t_{R}\right)$ at constant $R_{\Omega}$ is also shown.

that the greater the supersaturation rate, the greater the threshold supersaturation. The variation of $\Omega^{\text {th }}$ with $R_{\Omega}$ fits an empirical relationship that used to be familiar in the industrial crystallization literature (Putnis et al., 1995). The theoretical understanding of that correlation still requires deeper insight, however.

\section{Induction time and waiting time}

The induction time, i.e. the time $\left(t_{\mathrm{i}}\right)$ that elapses between the 'instantaneous' creation of supersaturation and the detection of nucleation, is another important concept in CNT. This time is related to the ability of the solution to remain supersaturated and, with some misgivings, can be considered an "experimental observable" (Kashchiev and van Rosmalen, 2003). In contrast, the waiting time $\left(t_{\mathrm{w}}\right)$ represents the total time involved in the transport-reaction process, from the beginning of the experiment to the moment at which $\Omega^{\text {th }}$ is reached and nucleation occurs. Following the comprehensive review by Kashchiev and van Rosmalen (2003), "when the appearance of the very first supernucleus in the solution volume is the event that brings the solution out of metastability", the induction time is given by:

$$
t_{\mathrm{i}}=1 / J V
$$

where $V$ is the solution volume. As Kashchiev and van Rosmalen (2003) noted, equation 8 implies setting $N=1$ at $t=t_{\mathrm{i}}$ in the expression $J=N / V t$. The approach in equation 8 is suitable only if we use experimental techniques that allow counting of $N$ or detection of the appearance of a single supernucleus. Although this point is frequently disregarded in the literature however, equation 8 is no longer valid when the $t_{\mathrm{i}}$ values are obtained by techniques that detect a mass of many nuclei.

\section{'We can see one'}

As discussed below, porous media are highly effective at suppressing nucleation. As a consequence, crystallization occurs at high supersaturation, and the nucleation density $(N / V)$ is typically very low. For this reason (and because of the hydrogel transparency), the gel technique provides certain research opportunities that nucleation in free solutions cannot provide. The low nucleation density allows us to detect and count the very first crystallites in the crystallization zone. In fact, certain typical papers on crystallization in gels (Prieto et al., 1990) report not only $t_{\mathrm{w}}$ and cz but also the number of crystallites observed. That feature makes equation 8 suitable for exploring the nucleation behaviour in this study.

Figure 4 shows an S-N-T diagram calculated for HON (solid line) using the parameters in Table 2. 


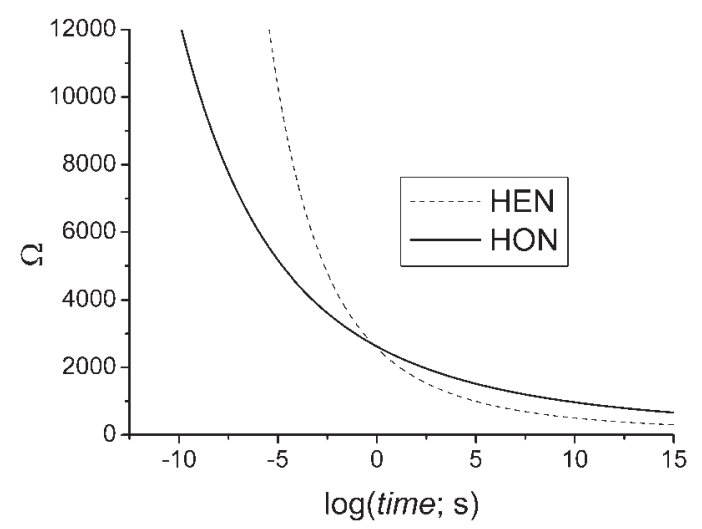

FIG. 4. S-N-T diagrams calculated for $\mathrm{HON}$ and HEN of baryte using the parameters in Table 2. The HEN diagram has been calculated using the number of active sites from experimental data by Garten and Head (1973).

The calculation has been performed considering the whole volume of the crystallization zone $(V=$ $\left.\pi r^{2} h=1.91 \mathrm{~cm}^{3}\right)$. The HEN diagram (dashed line) is somewhat speculative because parameters such as the concentration of active nucleation sites $\left(N_{0}\right)$ and $\Phi$ are difficult to estimate. Here, we have chosen $N_{0}=2.5 \times 10^{7} \mathrm{~cm}^{-3}$ from the data obtained by Garten and Head (1973) for baryte nucleation. Figure 5 shows the crystal counts obtained by these authors as a function of supersaturation. The strong increase above a certain supersaturation level is representative of $\mathrm{HON}$, whereas at a lower

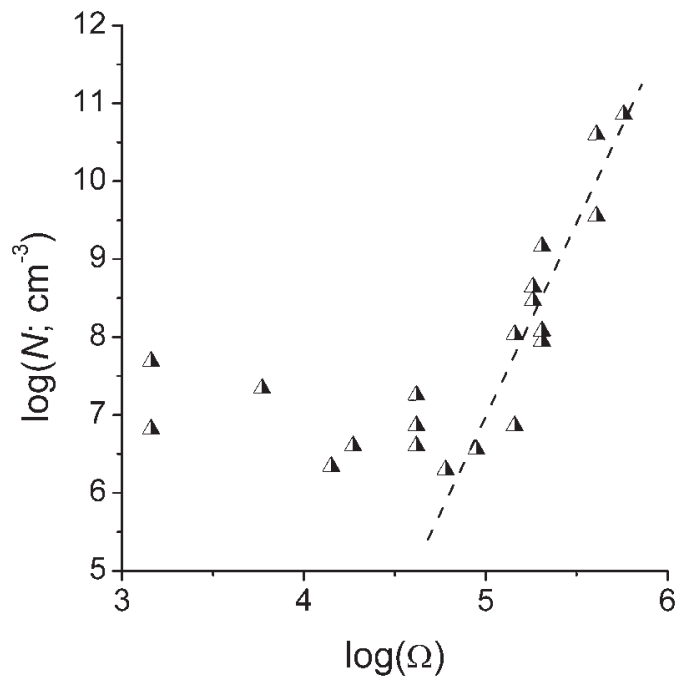

FIG. 5. Nucleation density of baryte in 'free' solutions as a function of supersaturation (recalculated from Garten and Head, 1973).

supersaturation, the number of nuclei is virtually independent of supersaturation and can be considered representative of the concentration of active HEN sites.

The diagram in Fig. 4 can be used to assess whether nucleation will or will not occur in an evolutionary system and at what degree of supersaturation. However, the diagram does not seem to work with gel experiments. As shown in

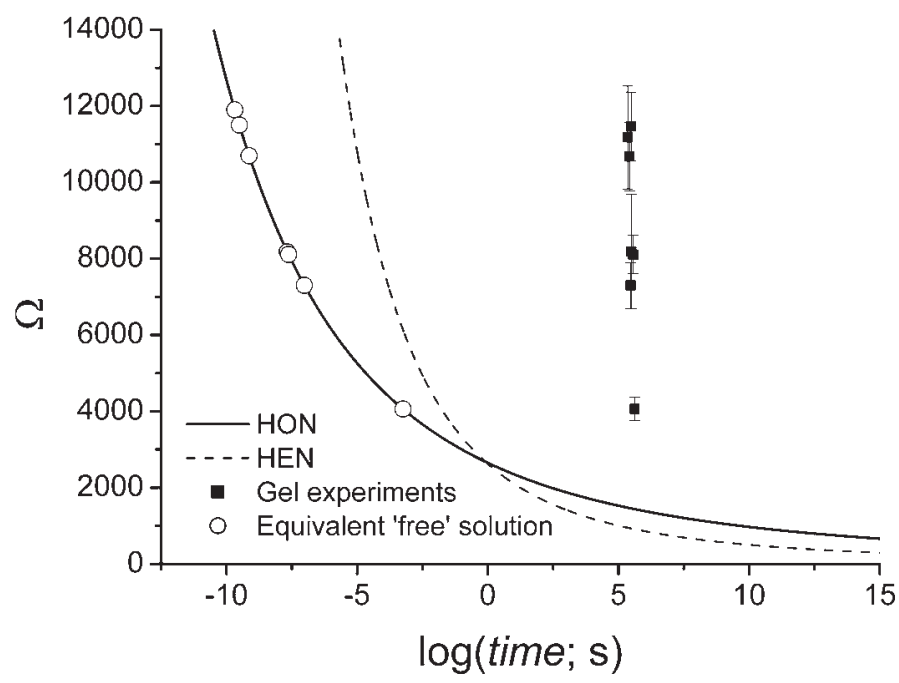

Fig. 6. Comparison of the experimental values of $\Omega^{\text {th }}$ with the theoretical S-N-T curves. The open circles show the induction times to be expected at equivalent supersaturations in $1.91 \mathrm{~cm}^{3}$ of 'free' solution. 
Fig. 6, the experimental $\Omega^{\text {th }}$ data points correspond to longer times in comparison with the theoretical nucleation curves. The experimental values of $\Omega^{\text {th }}$ have been plotted on the ordinate against $t_{\mathrm{R}}$ on the abscissa, i.e. considering a stationary supersaturation rate. The open circles show the induction times to be expected at equivalent supersaturation in $1.91 \mathrm{~cm}^{3}$ of 'free' solution. As observed, $t_{\mathrm{i}}$ and $t_{\mathrm{R}}$ differ by orders of magnitude.

\section{The pore size effect}

As seen in equation 8 , the induction time depends on the solution volume that we consider: the larger $V$, the shorter $t_{\mathrm{i}}$, in agreement with the probabilistic nature of nucleation. A problem arises because choosing the 'relevant' volume for nucleation-rate calculations is not always easy. The diagram in Fig. 4 was calculated by considering the whole volume of the crystallization zone, which does not seem to be the right choice. The fact that porous media are highly effective at suppressing nucleation lies at the core of this problem. During cluster formation, the solution vicinity becomes poor in solute, and the disappearance of a subcritical cluster is ensured if no exchange brings new growth units into its vicinity (Prieto et al., 2002). This effect is particularly important in fine porous media, where the solution is trapped in small pores connected by tortuous routes, and solute transport occurs by diffusion. Under these conditions, each single pore can be envisioned as a crystallization chamber, and we can use the pore size as the 'relevant' volume in equation 8.

Figure 7 shows two S-N-T diagrams calculated in this way for pore sizes of $1 \mu \mathrm{m}$ and $0.1 \mu \mathrm{m}$ (see Table 2). The experimental results plot between both curves, which demonstrate that the relevant volume in determining the nucleation behaviour is probably the pore volume and that the largest pores must be the preferential nucleation places. Although the HEN curve has not been represented, the high threshold supersaturation points towards an HON mechanism. The only exception occurs in experiment BRT-7. In this case, $\Omega^{\text {th }}$ and $R_{\Omega}$ are comparatively very small and a HEN mechanism could be expected. In counterdiffusion experiments the gel is said to be inert because it does not take part in the precipitation reaction. Nevertheless, in the same way as in free solutions, in real systems nucleation may occur on foreign nanoparticles or active centres present in either the polymeric framework or the interstitial solution. Anyway, Fig. 7 illustrates that S-N-T diagrams can be a suitable tool to assess HEN conditions in rocks, sediments, soils, etc., where the prediction of precipitation reactions is complicated not only by the porous nature of these media but also by their interaction with the precipitate (Stack et al., 2014).

Porous media are known to affect the transport of solutes through a diversity of mechanisms such as adsorption, absorption and precipitation

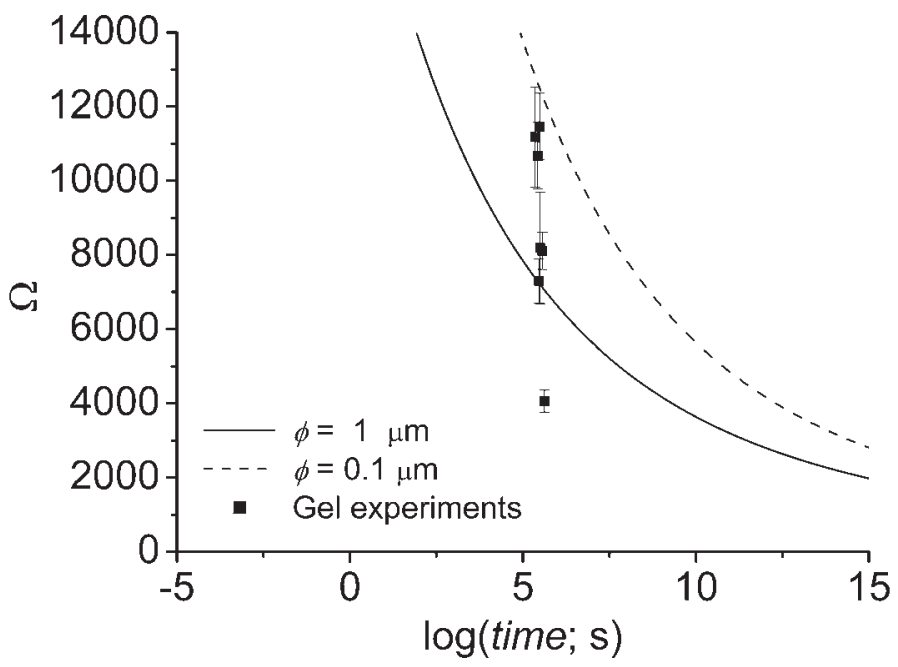

FIG. 7. S-N-T diagrams calculated for pore sizes of $1 \mu \mathrm{m}$ and $0.1 \mu \mathrm{m}$ (see Table 2). With the exception of experiment BRT-7, all the experimental $\Omega^{\text {th }}$ data plot between both curves. 
(Brown and Calas, 2013). However, the existence of a pore-size dependence of the metastability of supersaturated solutions is less known and not often considered in reactive-transport modeling. Such dependence usually results in inhibition of precipitation in nanopores and preference for the precipitation in macropores. Stack et al. (2014) studied the kinetics of precipitation of calcium carbonate in an amorphous-silica medium that contained two categories of pore sizes, macropores $(\varnothing>30 \mu \mathrm{m})$ and nanopores $(\varnothing=8-30 \mathrm{~nm})$. Those authors observed precipitation exclusively in the macropores and demonstrated how the interaction between substrate and precipitate is a controlling factor, with more "favorable" interactions allowing precipitation to occur in smaller pores. In fact, precipitation on nanopores can be enhanced using additives that increase the "favourability" of the substrate-precipitate interaction (Stack et al., 2014).

In the CNT framework, such 'favourability' can be expressed in terms of the HEN coefficient, $\Phi$. The smaller $\Phi$ is, the smaller the effective
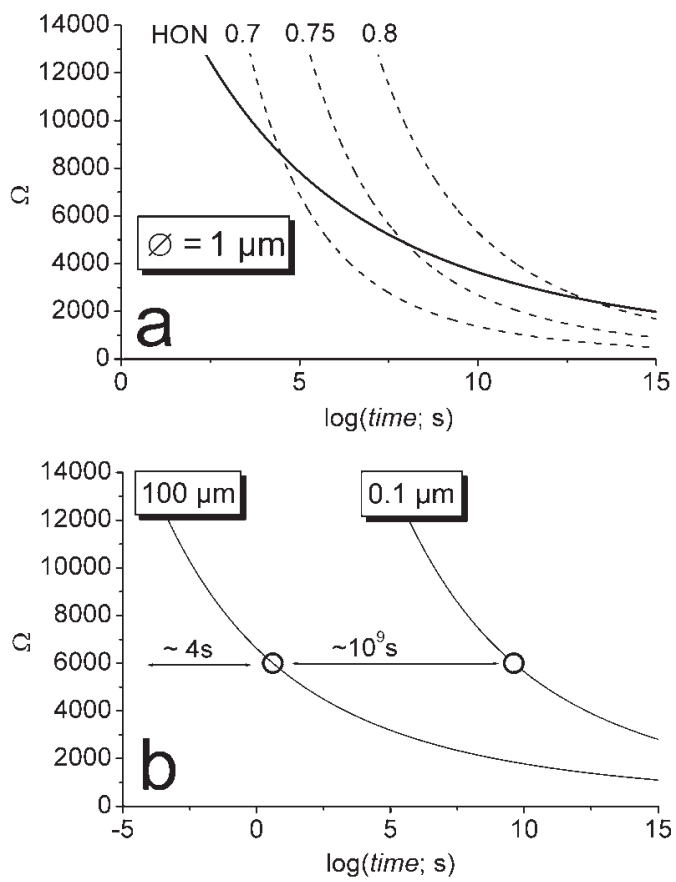

FIG. 8. (a) S-N-T diagrams calculated for a pore size of $1 \mu \mathrm{m}$ for homogeneous nucleation (HON) and heterogeneous nucleation with different degrees $(\Phi=0.7,0.75$, and 0.8 ) of 'favourability'. (b) Influence of the pore size on the HON behaviour in a porous medium with two categories $(100 \mu \mathrm{m}$ and $0.1 \mu \mathrm{m})$ of pores. interfacial tension, $\sigma_{\text {eff }}$ (see equation 7 ) and the larger the HEN probability. The effect is shown in Figure $8 a$ for the nucleation of baryte in $1 \mu \mathrm{m}$ sized pores. Figure $8 b$ illustrates the influence of the pore size in the case of $\mathrm{HON}$ in a porous medium with two categories $(100 \mu \mathrm{m}$ and $0.1 \mu \mathrm{m})$ of pores. As can be seen, for $\Omega=6000$ the induction time differs by nine orders of magnitude! Obviously, nucleation would occur in the $100 \mu \mathrm{m}$ pores. Only in case that the supply of reactants continued and $\Omega$ increased or kept constant for a long time, precipitation could eventually occur in the nanopores. These two examples show separately the main factors involved in the precipitation behaviour in porous media. In practice, the scenario will be a complex combination of both examples in which the interplay of nucleation mechanisms, pore-size effects, and supersaturation evolution will determine the final outcome.

\section{Conclusions}

S-N-T diagrams are shown to be a useful tool for interpreting and predicting nucleation behaviour in porous media. Such diagrams can be determined experimentally or estimated from CNTbased calculations. With this aim, the present author has adopted a pragmatic understanding of CNT in which the nucleation rate expression is imagined as a species of fitting function with the interfacial tension being the main fitting parameter. The method can be extended to a diversity of scenarios. The correct choice of the relevant volume for the studied system becomes clearer when one considers that nucleation in porous rocks is favoured in fractures and open spaces, where a faster supply of growth units is ensured.

\section{Acknowledgements}

This work was performed in the framework of the Marie Curie Network 'Geologic Carbon Storage' (European Commission, FP7-People-ITN-CO2REACT-317235). The author acknowledges Dr Cristina Ruiz-Agudo and two anonymous reviewers for their insightful comments. He also thanks the editors for their work and suggestions.

\section{References}

Baumgartner, J., Dey, A., Bomans, P.H.H., Le Coadou, C., Fratzl, P., Sommerdijk, N.A.J.M. and Faivre, D. (2013) Nucleation and growth of magnetite from solution. Nature Materials, 12, 310-314. 
Benning, L.G. and Waychunas, G.A. (2008) Nucleation, growth, and aggregation of mineral phases: mechanisms and kinetic controls. Pp. 259-332 in: Kinetics of Water-Rock Interaction (S.L. Brantley, J.D. Kubicki and A.F. White, editors). Springer-Verlag, Berlin.

Brown, G.E. and Calas, G. (2013) Mineral-aqueous solution interfaces and their impact on the environment. Geochemical Perspectiives, 1, 483-742.

De Yoreo, J.J. (2013) Crystal nucleation: More than one pathway. Nature Materials, 12, 284-285.

Demichelis, R., Raiteri, P., Gale, J.D., Quigley, D. and Gebauer, D. (2011) Stable prenucleation mineral clusters are liquid-like ionic polymers. Nature Communications, 2, 590.

Fernández-Díaz, L., Putnis, A., Prieto, M. and Putnis, C.V. (1996) The role of magnesium in the crystallization of calcite and aragonite in a porous medium. Journal of Sedimentary Research, 66, 482-491.

Fernández-Martínez, A., Hu, Y., Lee, B., Jun, Y. and Waychunas, G.A. (2013) In situ determination of interfacial energies between heterogeneously nucleated $\mathrm{CaCO}_{3}$ and quartz substrates: Thermodynamics of $\mathrm{CO}_{2}$ mineral trapping. Environmental Science \& Technology, 47, 102-109.

Forbes, T.Z., Radha, A.V. and Navrotsky, A. (2011) The energetics of nanophase calcite. Geochimica et Cosmochimica Acta, 75, 7893-7905.

Fritz, B. and Noguera, C. (2009) Mineral precipitation kinetics. Pp. 371-410 in: Thermodynamics and Kinetics of Water-Rock Interaction (E.H. Oelkers and J. Schott, editors). Reviews in Mineralogy and Geochemistry, 70, Mineralogical Society of America and the Geochemical Society, Chantilly, Virginia, USA.

Garten, V.A. and Head, L.B. (1973) Nucleation in salt solutions. Journal of the Chemical Society Faraday Transactions I, 69, 514-520.

Gebauer, D. and Cölfen, H. (2011) Prenucleation clusters and non-classical nucleation. Nano Today, 6, 564-584.

Gebauer, D., Völkel, A. and Cölfen, H. (2008) Stable pre-nucleation calcium carbonate clusters. Science, 322, 1819-1822.

Gebauer, D., Kellermeier, M., Gale, J.D., Bergström, L. and Cölfen, H. (2014) Pre-nucleation clusters as solute precursors in crystallization. Chemical Society Reviews, 43, 2348-2371.

Henisch, H.K. (1988) Crystals in Gels and Liesegang Rings. Cambridge University Press, Cambridge, UK.

Henisch, H.K. and García-Ruíz, J.M. (1986) Crystal growth in gels and Liesegang ring formation. I Diffusion relationships. Journal of Crystal Growth, 75, 195-202.

Kaschiev, D. (2000) Nucleation: Basic Theory with Applications. Butterworth-Heinemann, Oxford, UK.
Kashchiev, D. and van Rosmalen, G.M. (2003) Review: Nucleation in solutions revisited. Crystal Research and Technology, 38, 555-574.

Kowacz, M., Prieto, M. and Putnis, A. (2010) Kinetics of crystal nucleation in ionic solutions: Electrostatics and hydration forces. Geochimica et Cosmochimica Acta, 74, 469-481.

Lasaga, A.C. (1998) Kinetic Theory in the Earth Sciences. Princeton University Press, Princeton, New Jersey, USA.

Liu, X.Y. (1999) A new kinetic model for threedimensional heterogeneous nucleation. Journal of Chemical Physics, 111, 1628-1635.

Meldrum, F.C. and Sear, R.P. (2008) Now you see them. Science, 322, 1802-1803.

Nielsen, A.E. (1967) Nucleation in aqueous solution. Journal of Physical Chemistry of Solids, suppl. 1, 419-426.

Nindiyasari, F., Fernandez-Díaz, L., Griesshaber, L., Astilleros, J.M., Sanchez-Pastor, N. and Schmahl, W.W. (2014) Influence of gelatin hydrogel porosity on the crystallization of $\mathrm{CaCO}_{3}$. Crystal Growth \& Design, 14, 1531-1542.

Parkhurst, D.L. and Appelo, C.A.J. (2013) Description of input and examples for PHREEQC version 3 - A computer program for speciation, batch-reaction, one-dimensional transport, and inverse geochemical calculations. U.S. Geological Survey Techniques and Methods (http://pubs.usgs.gov/tm/06/a43), book 6, chap. A43.

Pouget, E.M., Bomans, P.H.H., Goos, J.A.C.M., Frederik, P.M., de With, G. and Sommerdijk, N.A.J.M. (2009) The initial stages of templatecontrolled $\mathrm{CaCO}_{3}$ formation revealed by cryo-TEM. Science, 323, 1455-1458.

Prieto, M., Putnis, A. and Fernández-Díaz, L. (1990) Factors controlling the kinetics of crystallization: supersaturation evolution in a porous medium. Application to barite crystallization. Geological Magazine, 127, 485-495.

Prieto, M., Fernández-González, A. and Martín-Díaz, R. (2002) Sorption of chromate ions diffusing through barite-hydrogel composites: Implications for the fate and transport of chromium in the environment. Geochimica et Cosmochimica Acta, 66, 783-795.

Prieto, M., Katsikopoulos, D. and Fernández-González, A. (2012) Interfacial tension and nucleation behaviour of minerals (revisited). Mineralogical Magazine, 76, 2251.

Putnis, A. (1992) Introduction to Mineral Sciences. Cambridge University Press, Cambridge, UK.

Putnis, A. and Mauthe, G. (2001) The effect of pore size on cementation in porous rocks. Geofluids, 1, 37-41.

Putnis, A., Prieto, M. and Fernández-Díaz, L. (1995) Fluid supersaturation and crystallization in porous media. Geological Magazine, 132, 1-13. 


\section{NUCLEATION AND SUPERSATURATION IN POROUS MEDIA}

Rodríguez-Blanco, J.D., Shaw, S. and Benning, L.G. (2011) The kinetics and mechanisms of amorphous calcium carbonate (ACC) crystallization to calcite, via vaterite. Nanoscale, 3, 265-271.

Rodríguez-Navarro, C., Doehne, E. and Sebastian, E. (2002) How does sodium sulfate crystallize? Implications for the decay and testing of building materials. Cement and Concrete Research, 30, 1527-1534.

Rodríguez-Ruiz, I., Veesler, S., Gómez-Morales, J., Delgado-López, J.M., Grauby, O., Hammadi, Z., Cadoni, N. and García-Ruiz, J.M. (2014) Transient calcium carbonate hexahydrate (ikaite) nucleated and stabilized in confined nano- and picovolumes. Crystal Growth \& Design, 14, 792-802.

Sancho-Tomas, M., Fermani, S., Duran-Olivencia, M.A., Otalora, F., Gomez-Morales, J., Falini, G. and García-Ruiz, J.M. (2013) Influence of charged polypeptides on nucleation and growth of $\mathrm{CaCO}_{3}$ evaluated by counter-diffusion experiments. Crystal Growth \& Design, 13, 3884-3891.

Sangwal, K. (2007) Additives and Crystallization Processes: from Fundamentals to Applications. Wiley \& Sons, Oxford, UK.

Shtukenberg, A.G., Astilleros, J.M. and Putnis, A. (2005) Nanoscale observations of the epitaxial growth of hashemite on barite (001). Surface Science, 590, 212-223.

Söhnel, O. (1982) Electrolyte crystal-aqueous solution interfacial tensions from crystallization data. Journal of Crystal Growth, 57, 101-108.
Stack, A.G., Fernandez-Martinez, A., Allard, L.F., Banuelos, J.L., Rother, G., Anovitz, L.M., Cole, D.R. and Waychunas, G.A. (2014) Pore-sizedependent calcium carbonate precipitation controlled by surface chemistry. Environmental Science \& Technology, 48, 6177-6183.

Vekilov, P.G. (2010) Nucleation. Crystal Growth \& Design, 10, 5007-5019.

Walton, A.G. (1969) Nucleation in liquids and solutions. Pp. 225-307 in: Nucleation (A.C. Zettlemoyer, editor). Marcel Dekker, New York.

Wang, L., Li, S., Ruiz-Agudo, E., Putnis, C.V. and Putnis, A. (2012) Posner's cluster revisited: direct imaging of nucleation and growth of nanoscale calcium phosphate clusters at the calcite-water interface. CrystEngComm, 14, 6252-6256.

Wu, W. and Nancollas, G.H. (1999) Determination of interfacial tension form crystallization and dissolution data: a comparison with other methods. Advances in Colloid and Interface Science, 79, 229-279.

Yi, P. and Rutledge, G.C. (2012) Molecular origins of homogeneous crystal nucleation. Annual Review of Chemical and Biomolecular Engineering, 3, 157-182.

Yuwono, V.M., Burrows, N.D., Soltis, J.A. and Penn, R.L. (2010) Oriented aggregation: formation and transformation of mesocrystal intermediates revealed. Journal of the American Chemical Society, 132, 2163-2165. 